\title{
La tutoría como estrategia pedagógica para el desarrollo de competencias de investigación en posgrado
}

\section{Tutorship as a Pedagogical Strategy for the Development of Postgraduate Research Competencies}

\author{
AlejandroCruzata-Martínez* (iD Universidad San Ignacio de Loyola, Lima, Perú. \\ ORCID: https://orcid.org/0000-0003-0104-0496 \\ Roberto Bellido García Universidad San Ignacio de Loyola, Lima,Perú. \\ ORCID: https://orcid.org/0000-0002-1417-3477 \\ Miriam Velázquez-Tejeda Universidad San Ignacio de Loyola, Lima, Perú. \\ ORCID: https://orcid.org/0000-0002-6245-6690 \\ Joel Alhuay-Quispe (iD) Universidad San Ignacio de Loyola, Lima, Perú \\ ORCID: https://orcid.org/0000-0002-1903-4687
}

Recibido 18-07-18 Revisado 30-07-18 Aprobado 17-10-18 En línea 18-10-18

*Correspondencia

Email: acruzatam@usil.edu.pe

\section{Citar como:}

Cruzata-Martínez, A., Bellido, R., VelázquezTejeda, M., \& Alhuay-Quispe, J. (2018). La tutoría como estrategia pedagógica para el desarrollo de competencias de investigación en posgrado. Propósitos y Representaciones, 6(2), 09-62. Doi: http://dx.doi.org/10.20511/pyr2018.v6n2.252

(C) Universidad San Ignacio de Loyola, Vicerrectorado de Investigación, 2018

(cc)BY-NC-ND Este artículo se distribuye bajo licencia CC BY-NC-ND 4.0 Internacional (http://creativecommons.org/licenses/by-nc-nd/4.0/). 


\section{Resumen}

La producción de conocimiento es un elemento esencial en las escuelas de posgrado y se considera que este proceso está muy avanzado cuando el estudiante culmina y sustenta su tesis de investigación. Sin embargo, una cantidad de estudiantes no logra desarrollar las competencias investigativas y culminar la tesis. El objetivo de esta propuesta es precisar los roles y las competencias que demanda el tutor de posgrado para lograr realizar un acompañamiento y mediación en los estudiantes de posgrado, potenciando la articulación de la tutoría universitaria y la investigación científica. Se propone una propuesta que permita fortalecer las competencias investigativas en los estudiantes de posgrado por medio de la acción tutorial. La propuesta se basa en el modelo integrador de la tutoría desarrollado por Cruz, García y Abreu (2006), dirigidos a los tutores y estudiantes que se encuentran desarrollando sus tesis de investigación en la escuela de posgrado.

Palabras clave: Competencias investigativas, tutoría, evaluación del desempeño. 


\section{Summary}

The production of knowledge is an essential element in graduate schools and it is considered that this process is very advanced when the student completes and defends his research thesis. However, a number of students fails to develop research skills and complete their thesis. The objective of this proposal is to specify the roles and competencies required by the postgraduate tutor to achieve accompaniment and mediation in postgraduate students, promoting the organization of university tutorship and scientific research. A proposal that allows the strengthening of the research competencies in postgraduate students by means of the tutorship is raised. The proposal is based on the integrating model of the tutorship developed by Cruz, García and Abreu (2006), aimed at tutors and students who are developing their research theses at the graduate school.

Keywords: Research competencies, tutorship, performance evaluation. 


\section{Introducción}

La universidad científica, concebida en la Universidad de Berlín, a inicios del siglo XIX, por los hermanos Humboldt y otros, tenía como uno de sus soportes fundamentales, el doctorado de investigación, basado en un tutor con gran experiencia investigativa, que asignaba una tesis a su discípulo, y lo guiaba hasta su defensa. Ese modelo, ha mostrado su fortaleza, y todavía está presente en la gran mayoría de los programas de maestrías y doctorados que se realizan en el mundo.

No obstante, hoy existen un conjunto de factores que influyen en el cuestionamiento de dicho modelo como condición indispensable para garantizar la calidad de un trabajo final de posgrado dirigido a la producción de nuevos conocimientos y a la formación de investigadores:

- Muchas de las nuevas capacidades que demanda la formación del profesional, no son promovidas por este modelo.

- Los cambios que se han producido en la forma de generación de nuevos conocimientos, van de diversas maneras en el sentido contrario a los métodos de formación del modelo tutelar clásico.

- La extensión de los programas académicos de posgrado, restringidos antes a la preparación para la investigación básica y hoy ampliados al perfeccionamiento de todas las profesiones, cada una con sus propias características.

- El crecimiento extraordinario de la cantidad de graduados de la educación superior que genera una alta demanda de estudios de posgrado.

Para satisfacer necesidades del desarrollo local, resulta indispensable conceptualizar también un modelo de tutoría muy pertinente al propósito de la formación de investigadores en los campos de las ciencias exactas, naturales, sociales y otras ramas de la investigación básica. 
Un elemento muy importante que tomar en cuenta en este tipo de programa es que el mismo no compromete a la institución con una titulación final, sino que su propósito es brindar, dentro del concepto de educación permanente o durante toda la vida, la oportunidad o el derecho a titularse dentro de un programa académico de posgrado, partiendo fundamentalmente del esfuerzo personal del estudiante. Por eso, la institución educativa debe crear las condiciones para facilitar, dentro de sus posibilidades, la labor de tutoría con métodos diferentes a los aceptados dentro de los programas académicos tradicionales.

Los programas académicos de posgrado requieren de un sistema de tutoría que permita la atención a estudiantes, frente a esta situación surgen las siguientes interrogantes ¿Cómo desarrollar las competencias investigativas en posgrado mediante el trabajo tutorial? ¿Cuáles son los roles que debe tener el tutor para desarrollar competencias investigativas en los estudiantes de posgrado?, ¿Qué competencias demanda el tutor para desarrollar las competencias investigativas?

En ese sentido, los objetivos de la investigación son:

- Proponer los roles de los tutores para desarrollar las competencias investigativas en los estudiantes de posgrado.

- Explicar las competencias que demanda desarrollar el tutor en posgrado.

\section{Planteamiento conceptual.}

\section{Competencias investigativas en posgrado.}

Uno de los problemas más recurrentes en la práctica social ha sido cómo evaluar la calidad de los egresados de la educación superior en la búsqueda de la eficiencia de sus resultados en el contexto donde deben desempeñarse. Términos como inteligencia, conocimientos, destrezas, capacidades, habilidades, creatividad, talento, en las últimas décadas se les ha sumado 
valores, significados personales, se han empleado para identificar la calidad. Una calidad que se evalúa al comparar los resultados que obtiene el egresado con el modelo social ideal de la excelencia y que depende en lo fundamental del sujeto individual. Una de las propuestas contemporáneas que intenta superar esta contradicción define el término competencias.

A pesar de tantos intentos, no existe una definición única del término, y cada autor enfatiza alguna cualidad de acuerdo con sus intenciones o prácticas profesionales. La mayoría de las definiciones comprenden las competencias como unidades de actuación que expresan lo que una persona debe saber y poder hacer para desarrollar y mantener un nivel de desempeño eficiente en su labor. Incluye aspectos cognitivos, afectivos, conductuales y de experiencia.

Las competencias profesionales son aquellas que permiten al individuo solucionar los problemas inherentes al objeto de su profesión en un contexto laboral específico, en correspondencia con las funciones, tareas y cualidades profesionales que responden a las demandas del desarrollo social.

Cada profesión y cada profesional, en dependencia del objeto de la profesión, del rol social que se le asigne, así como de las funciones y tareas que le competen, debe desarrollar aquellas competencias que le son inherentes a su actividad, lo que le permite el logro de desempeños exitosos, eficientes.

"Incorporar el concepto de competencias a la práctica educativa, significa que el resultado va a estar ligado a acciones concretas, no como efecto de un aprendizaje tradicional, sino un aprendizaje donde se acrecienten las capacidades humanas mediante el desarrollo integrado de las dimensiones cognoscitivas de la personalidad, en el cual la búsqueda, la indagación, el uso de métodos científicos, caracterice la solución de los problemas" (Fuentes \& Cruz, 2001, p. 2).

Las competencias investigativas que exige la escuela de posgrado son:

- Comunicación integral: 
Conoce y utiliza los rasgos distintivos del lenguaje culto y académico para comunicar de forma oral y escrita la información pertinente para su desempeño personal y profesional.

- Investigación Científica:

Desarrolla procesos de investigación a través del método científico general para diseñar y aplicar propuestas que den tratamiento o soluciones a distintos temas con carácter aplicado.

- Gestión de recursos:

Gestiona desde los procesos de investigación con carácter interdisciplinario los recursos necesarios para insertarse en los diferentes espacios competitivos que le permiten su desarrollo profesional óptimo.

- Desarrollo humano:

Demuestra y hace uso de los conocimientos previos para desarrollar sus habilidades profesionales que le permiten contribuir e intervenir en los procesos de reconstrucción o construcción de los distintos contextos sociales en los que se desarrolla.

\section{El tutor y la tutoría.}

Coromoto (2007) plantea que etimológicamente, tutor significa: "maestro, amigo, guía, acompañante, defensor, protector, guardián, director, y sostén”. Martínez (2005) define que "el tutor es un asesor, un mentor, un guía, y a la vez, un supervisor. Su trabajo es muy delicado porque debe saber qué y cómo debe orientar a sus tutorados sin suplantar su gestión y logrando su máxima independencia. O sea, para el desarrollo de su labor debe apoyarse no sólo en el conocimiento del área de estudios correspondiente, sino en una serie de regularidades de carácter psicológico y pedagógico que darán rigor a su trabajo, sobre todo las relacionadas con el carácter creador de su gestión".

Para Sánchez (2001), el tutor, en la Educación Abierta y a Distancia, tiene tres funciones: orientadora, docente y motivadora. En la primera familiariza al estudiante con la modalidad de estudio, en la segunda debe garantizarle 
los recursos necesarios y condiciones para la transmisión de conocimientos y en la tercera debe propiciar que el estudiante descubra, sus aspiraciones, sus intereses y motivaciones que lo puedan conducir a una actuación más responsable ante sus deberes hasta alcanzar las metas trazadas e incluso a trazarse nuevas metas cualitativamente superiores.

En este sentido, en la actualidad, la tutoría se lleva a cabo en diversas modalidades educativas, en numerosas áreas del conocimiento y en distintos niveles educativos (licenciatura, posgrado), por lo que el concepto de tutor y la función difieren en sus aspectos operativos.

- La enseñanza tutoral

Ésta, al nivel de licenciatura, se entiende como: ...una modalidad de instrucción en la que un maestro (tutor) proporciona educación personalizada a un alumno o a un grupo reducido de estudiantes. Generalmente se adopta como medida emergente o complementaria para estudiantes con dificultades para seguir los cursos convencionales (Latapí 1988).

Así, en los estudios de licenciatura, se ha considerado tradicionalmente como equivalente a la dirección de tesis y, más recientemente, se ha añadido el concepto de tutoría como acompañamiento durante el proceso escolar (ANUIES, 2000). De otra parte, en el postgrado, se privilegia la productividad científica de los tutores como condición suficiente para guiar el proceso formativo del tutorado, el cual suele centrarse en la investigación de un tema altamente especializado, frecuentemente en detrimento de una visión panorámica y equilibrada del campo.

La tutoría tradicional prepara a los tutorados para el éxito académico o escolar, descuidando el desarrollo de capacidades para contender con la complejidad y la incertidumbre inherente a la sociedad del conocimiento (Stehr, 1994). La sociedad actual demanda individuos capaces de actuar en los ambientes reales y dinámicos de la actividad profesional misma, formados para trabajar con problemas mal definidos, que involucran múltiples variables y no respetan fronteras disciplinarias. 
Según Ceja, Vengas y Armenta (1988) el tutor debe asumir una actitud de facilitador y guía de la interacción y el trabajo de los tutorados y asimismo, reunir las siguientes características:

- Dominio de la disciplina y de su campo profesional.

- Conocer el nivel de formación de los tutorados y las competencias que han desarrollado.

- Empatía.

- Capacitación socio-psicopedagógica.

- Estar dispuesto a brindar el apoyo necesario, en el momento preciso, de acuerdo a las necesidades del estudiante.

- Ser mediador a fin de que el estudiante adopte sus propias ideas, en las relaciones que establece el tutorado con los usuarios de los servicios y con otros colegas.

- Reconocerse a sí mismo como un profesional que no tiene todas las respuestas.

- Ser un facilitador y modelo en el desarrollo de conocimientos, habilidades profesionales y comunicación de valores y ética profesional.

Por todo lo anterior, se puede señalar que la tutoría se conduce de tutor a estudianteo a un grupo de estudiantes, que puede ser presencial o a distancia, y que apoya a los estudiantes en la construcción del conocimiento y en la preparación de exámenes. Sin embargo, lo más importante de este proceso es la participación activa del tutorado en su proceso de aprendizaje.

El tutor es un apoyo, un guía y un facilitador del desarrollo de la capacidad creativa y crítica del estudiante. 


\section{El tutor de posgrado.}

Si bien el Reglamento de Estudios de Posgrado señala los requisitos académicos para que un profesor de carrera de tiempo completo sea acreditado como tutor, así como las responsabilidades generales, no se describen las competencias que deben poseer los tutores que participan en este nivel de estudios.

Esta circunstancia dificulta la selección de nuevos tutores, el desarrollo de programas de actualización y, en forma particular, la evaluación del desempeño de los tutores y su papel en el logro de los objetivos y metas propuestos por cada programa.

El tutor debe reunir una serie de competencias que le permitan el logro de los objetivos académicos; por tal motivo resulta indispensable identificar aquellas características que se requieren para ser un tutor competente.

El concepto operativo de competencias del tutor fue definido como: un conjunto de conocimientos, habilidades, actitudes y valores que posee el tutor y que, relacionados entre sí permiten el desempeño satisfactorio de las actividades y funciones en el proceso educativo, según los indicadores y estándares establecidos, en el contexto educativo, con la finalidad de que el estudiante, al concluir sus estudios, se incorpore con ventajas a la sociedad.

Con base en el análisis de las funciones y roles de los tutores analizados en el capítulo anterior, así como de los conocimientos, habilidades, actitudes y valores en su conjunto, validados por los comités académicos de los programas de postgrado, se realizó una clasificación teórica de las competencias académicas identificadas por tutores expertos, las mismas que se presentan en el próximo epígrafe.

El tutor de postgrado ha sido conceptualizado como:

- Figura indispensable para la mejor consecución de la excelencia en los estudios de postgrado (Sarukhán, 1988).

- Componente sine qua non de cualquier programa de posgrado. 
- Debe ser un guía que oriente debidamente el proceso de aprendizaje del tutorado, para lo cual debe adaptar el sistema académico a las características particulares del estudiante [...] e tutor forma cuadros académicos de alto nivel intelectual [...] el tutor debe ser un experto reconocido en su área de conocimiento, que se encuentre desempeñando activamente las tareas de investigación y docencia directamente relacionadas con el objeto de la tutoría (Garritz, \& López, 1989).

El tutor de postgrado debe cumplir con la misión de formar a las nuevas generaciones, capacitarlas para llevar a cabo investigación relevante, de alto nivel académico y compromiso social, que contribuya a la solución de problemas nacionales y, al mismo tiempo, fortalezca el sistema educativo nacional en todos sus niveles.

En el postgrado, el proceso educativo está orientado a la fundamentación, diseño y ejecución de una investigación; es decir, a la generación de nuevos conocimientos.

En México, el Instituto Politécnico Nacional, refiere las siguientes responsabilidades de los tutores de postgrado:

- Orientar al estudiante en el desarrollo de su programa de actividades.

- Ayudar al estudiante desde el inicio y hasta el término de su investigación.

- Prever la carga académica, las actividades de investigación y los seminarios que le ayuden a su formación.

- Apoyar el desarrollo de competencias personales y transversales del doctorado: responsabilidad, razonamiento, comunicación y trabajo en equipo.

- Compartir con el doctorando sus métodos, técnicas y experiencias.

- Retroalimentar y evaluar los avances del trabajo del estudiante. 
- Apoyar en la elaboración de la tesis de grado del doctorando para que culmine sus estudios.

Las prácticas de tutoría pueden ser múltiples y variadas, en especial en el nivel de maestría, por lo que en este trabajo se hará hincapié en la tutoría de maestría y doctorado con orientación a la formación de investigadores.

Existen diversas opiniones sobre la importancia y finalidad de la tutoría a nivel postgrado, entre las que destacan:

- Consiste en una relación obligatoria de un cierto número de horas a la semana o al mes.

- Debe ser, fundamentalmente, la posición de una persona que analiza escenarios, que propone opciones, que sugiere posibilidades de acción y que le deja al estudiante la toma de decisiones.

- Constituye un vínculo necesario entre la investigación y la docencia.

- El sistema tutoral para los maestrantes se define como la atención personalizada para el diseño, formulación y elaboración del proyecto de investigación

Garritzy López(1989) indica que: ...la tutoría ofrece una alternativa para construir un espacio en el cual se propicie el desarrollo de las potencialidades de los futuros investigadores, ya que posibilita una relación estrecha entre el maestro y el estudiante, al concebirse aquél como un guía que orienta a éste en el transcurso de sus actividades académicas.

Como se puede apreciar, la tutoría se ha convertido en una de las mejores estrategias para elevar la calidad de la educación de postgrado, especialmente en los niveles de maestría y doctorado. Al respecto, Garritz y López (1989) señalan que, para el sano funcionamiento de la maestría o el doctorado, la investigación es un elemento indispensable" y agrega que esta es su esencia misma.

De esta manera, la tutoría en el postgrado es una relación bipersonal (tutor-estudiante), en la que ambos participan en la creación de nuevos 
conocimientos y en el análisis de la información disponible; estos aspectos distinguen a la tutoría del postgrado de las modalidades y niveles educativos, ya que su fin último está plasmado en el diseño, desarrollo y análisis de una investigación original, producto que conjuga el aprendizaje del estudiante y el desarrollo de ciertas potencialidades propias del acto investigativo.

En la tutoría, como relación bipersonal o interpersonal, participa tanto el estudiante como el tutor, entre ambos se genera un sinnúmero de intercambios, ya sea académicos, personales, en diversos escenarios y contextos complejos. De todo esto depende, en gran medida, el éxito o fracaso académico (eficiencia terminal) del estudiante de postgrado. Lo anterior implica la necesidad de estudiar a cada uno de los elementos de este proceso por separado.

La tutoría, como proceso, permite que el estudiante obtenga una formación suficiente para lograr éxito como profesional, investigador y docente; para la toma de decisiones importantes en su vida académica; y para el desarrollo de habilidades, destrezas, actitudes y valores necesarios en el ámbito académico, así como en los aspectos personales y sociales.

Es importante subrayar que el prestigio que alcanza un programa de postgrado depende, esencialmente, de la calidad de sus tutores y de la medida en que sus egresados cumplen a plenitud con los propósitos de la educación del postgrado: responder a las necesidades sociales, presentes y futuras.

\section{Roles de la tutoría: una explicación necesaria.}

El término rol, ampliamente utilizado en la literatura sociológica y psicológica, proviene del francés role, que significa papel desempeñado por un actor (Diccionario Enciclopédico, 1953, p. 601). Con esta acepción pasó, como galicismo, primero al inglés y más tarde al castellano, donde equivale al papel o parte dentro de un libreto y se extiende a los significados de misión, encomienda, compromiso, etc. (Diccionario Enciclopédico, 1953, p. 908). 
En el terreno de las Ciencias Sociales, particularmente en la Sociología, el concepto de rol tiene una connotación especial, a partir de los trabajos de Talcott Parsons (1945, p. 283), que identificó con este término el papel específico que ocupa un sujeto dentro de un sistema de relaciones humanas.

De hecho, según la teoría parsoniana, el mismo sujeto es un actor que desempeña diversos roles sociales que se manifiestan a través de funciones, pudiendo ser manifiestas o latentes. Las primeras serán aquellas funciones aceptadas y reconocidas por los actores, las segundas son aquellas que no tienen aceptación o reconocimiento, que pueden conducir a comportamientos patológicos, definidos como “disfuncionales" (Merton, 1973, p. 165).

El aporte de Parsons que dio lugar a la llamada "teoría de los roles" es parte integrante del conocimiento sociológico contemporáneo y, por tanto, de la construcción epistémica de la Sociología de la Educación, en lo que mucho tuvo que ver el desarrollo posterior de dicha teoría, realizado por Merton (1973, p. 166).

\section{Resultados}

A partir de lo establecido se asumen los roles del tutor, propuestos por Cruz, García y Abreu (2006) en su trabajo Modelo integrador de la tutoría. Estos autores identifican ocho roles que coexisten simultáneamente y se dividen solo para su estudio en roles que conforman el eje vertical de la tutoría y el eje horizontal.

Existen dos roles centrales que configuran un eje vertical, los cuales organizan a la tutoría en su conjunto, a saber: formación investigativa y formación profesional, que abarca el rol docente, socializador, entrenamiento (coaching), consejería académica, patrocinador y apoyo psicosocial. La propuesta se concentra en:

- El rol del tutor para la formación en investigación. 
- Las competencias que debe poseer el tutor para la formación en investigación.

- Como evaluar el rol del tutor.

Formación en investigación, que tiene dos vertientes: primero sustentar la práctica profesional en la mejor evidencia científica disponible, y segundo, generar nuevo conocimiento, siendo ésta una función más propia del postgrado.

a) La tutoría debe promover una práctica profesional sustentada en la evidencia científica disponible, para lo cual el educando debe aprender a identificar problemas y estructurar preguntas relevantes; buscar, localizar, recuperar y analizar críticamente información biblio-hemerográfica relacionada con asuntos profesionales, para que postule posibles soluciones y reflexione sobre su acción con conocimiento de causa.

Además, se deberá formar a los tutorados para que tengan capacidad de evaluar la metodología utilizada en investigaciones recuperadas, con el fin de apreciar

La validez de los resultados y juzgar si contribuyen a aclarar o resolver problemas profesionales, considerando enfoques multi o transdisciplinarios relevantes para la práctica profesional, así como ser capaces de proponer proyectos de investigación para llenar los vacíos de conocimiento.

La guía de la tutoría permitirá a los educandos organizar el conocimiento relevante para elaborar modelos conceptuales que permitan apreciar la complejidad de los problemas y postulen soluciones apoyadas en la evidencia científica disponible.

b) La tutoría debe preparar para evaluar, con rigor metodológico, las intervenciones de carácter profesional, debiendo elaborar modelos conceptuales que consideren las variables involucradas y los posibles factores de confusión; identificando diseños de investigación idóneos, así como la factibilidad y aspectos éticos de la evaluación. 
Los tutorados serán capaces de operar variables, seleccionar o desarrollar instrumentos, verificar la calidad de sus datos, realizar análisis estadísticos, interpretar y contrastar sus resultados con otros estudios similares, para obtener conclusiones válidas y confiables que contribuyan a mejorar su desempeño profesional, permitiéndoles valorar la posible generalización de sus resultados y su potencial transferencia a otros contextos.

Para quienes se forman para realizar investigación orientada a generar nuevos conocimientos conviene identificar, por lo menos, tres grandes etapas: planteamiento, desarrollo y conclusión.

\section{Planteamiento de la investigación.}

Durante esta etapa, la tutoría dirige al tutorado en el proceso de búsqueda, localización, recuperación y evaluación de la información relevante para acotar el problema de investigación.

Lo orienta en la revisión crítica y multidisciplinaria del material recuperado para sistematizarlo y elaborar un estado del arte que le permita visualizar los problemas en su complejidad, considerando la multicausalidad, el posible orden temporal y la dirección de las relaciones entre variables, así como el carácter dinámico de los fenómenos; configurando un marco de referencia general sobre el fenómeno a estudiar que permita identificar potenciales problemas de investigación de carácter científico o profesional, valorando tanto su relevancia científica como su trascendencia social.

Se asesora al estudiante en la selección del problema que servirá para elaborar la investigación y, en su caso, la tesis de grado. Se le guía para que construya modelos conceptuales sustentados en el conocimiento previo, buscando capacidad explicativa y consistencia interna, a fin de identificar variables o factores involucrados con el problema, ordenados temporalmente para generar hipótesis.

Lo orienta en la selección de estrategias metodológicas que permitan someter a prueba las hipótesis, controlar los posibles factores de confusión 
para otorgar validez al estudio, evitar el reduccionismo y juzgar la factibilidad y viabilidad de la investigación.

Asesorar en la selección de las técnicas idóneas para estructurar el plan de análisis de los datos, de conformidad con el diseño y las preguntas de investigación, lo que deberá plasmarse en el protocolo de investigación y, en su caso, presentarlo y defenderlo ante los órganos académicos establecidos para evaluar la calidad de la investigación propuesta y, cuando se requiera, se le orientará para obtener la aprobación de comités de investigación y de ética.

\section{Desarrollo de la investigación.}

Esta etapa se orienta a que el tutorado ejecute la investigación, ayudándolo a montar técnicas de laboratorio o bien a realizar estudios piloto cuando sean necesarios. Supervisa la obtención, calidad y organización de los datos. Auxilia para que pueda responder ante imprevistos, analizando posibles formas de abordarlos y orienta al tutorado en la toma de decisiones para realizar ajustes indispensables al proyecto original.

Lo asiste en la presentación de sus avances en distintos foros académicos y en la valoración e integración, cuando corresponda, de las observaciones emitidas por los comités evaluadores.

\section{Conclusión de la investigación.}

La tutoría auxilia al tutorado en el análisis de los datos e interpretación de los resultados, contrastándolos con los antecedentes teóricos y empíricos relacionados, propiciando que identifique los alcances y limitaciones del estudio, así como nuevas hipótesis, otros problemas de investigación y posibles aplicaciones. Por último, lo asiste en la selección de las revistas o casas editoriales idóneas para la publicación de resultados, auxiliándolo en la redacción de conformidad con los lineamientos de los editores; así como en la realización de ajustes pertinentes y en dar respuesta a las sugerencias de los árbitros. 
A lo largo del proceso de investigación, la tutoría fomentará que el tutorado articule críticamente el corpus de conocimiento del campo de estudio con la praxis y resultados de su proyecto de investigación.

La formación en investigación deberá contribuir a exponer al tutorado a una pluralidad de problemas profesionales y proyectos de investigación para que sea capaz de visualizar la complejidad del campo, identifique y seleccione el conocimiento científico necesario para su práctica profesional o bien para plantear nuevos proyectos de investigación de manera independiente y autorregulada.

\section{Competencias básicas que debe poseer el tutor.}

\section{Competencia cognoscitiva.}

Formación psicológica que le permite al tutor la actualización sistemática y superación permanente, que posibilita su desarrollo constante para orientar contenidos amplios, profundos, actualizados y esenciales. Se manifiesta en la capacidad cognoscitiva.

Se pone de manifiesto en la búsqueda, registro, estudio y actualización sistemática de la cultura para el dominio del contenido relacionado con su labor profesional como dimensión del modo de actuación profesional, se identifica por las acciones que realiza para obtener la información de diversas fuentes, su procesamiento y utilización en función de las potencialidades formativas que poseen.

Es también contenido a estudiar y dominar la caracterización integral de los tutorados desde todas sus dimensiones, cognitiva, afectiva, actitudinal, que deben ser utilizados en la dirección del proceso de educación. Para tener éxito en esta labor de búsqueda, estudio y dominio del contenido, es necesario el desarrollo de esta capacidad expresada en las habilidades y hábitos de localización, procesamiento, registro e interpretación de la información de las diferentes fuentes, así como la motivación y disposición para hacerlo de 
manera sistemática y por diferentes vías, incluyendo la autosuperación y la superación académica.

Ese modo de actuar condiciona la formación de una cualidad de la personalidad del docente que lo debe caracterizar como una persona culta, estudiosa y actualizada.

En esta competencia se destacan los componentes siguientes:

1) Los conocimientos sobre la información.

- Dominio sobre la teoría de la información.

- Dominio de las características de las diferentes fuentes de información.

- Dominio sobre las vías y los métodos para obtener la información.

- Dominio de cómo funcionan los procesos cognoscitivos y los componentes de la competencia para su autorregulación, autoperfeccionamiento y autoevaluación.

2) Las habilidades intelectuales, comunicativas, profesionales y hábitos de búsqueda, localización, procesamiento, registro e interpretación de la información de las diferentes fuentes, tales como:

- Saber orientarse en centros de información a través de los ficheros y otras vías automatizadas.

- Saber hacer búsquedas bibliográficas sobre un tema Saber procesar y registrar la información en resúmenes, fichas de diferentes tipos, elaborar esquemas, gráficos, informes, mapas conceptuales, etc.

- Saber interpretar la información haciendo uso crítico de la misma para fundamentar sus ideas, a través de todo el sistema de habilidades lógicas y los procesos del pensamiento.

- Saber producir información en forma de artículos, noticias, medios de enseñanza, libros, materiales docentes, etc. 
- Saber planificar la actividad científico-investigativa.

- Saber ejecutar el proceso de investigación.

- Saber comunicar los resultados de la actividad científica.

Operaciones fundamentales que integran la capacidad cognoscitiva:

a) Búsqueda, estudio sistemático, procesamiento y registro de la información actualizada sobre el contenido de las ciencias relacionadas con la asignatura, el área del conocimiento, el grado y la enseñanza en la que desarrolla su labor.

b) Búsqueda, estudio sistemático, procesamiento y registro del contenido de la didáctica para su labor de educación desde una concepción integradora y contextualizada.

c) Búsqueda, estudio sistemático, procesamiento y registro de la información sobre los fundamentos filosóficos, psicológicos, sociológicos, pedagógicos y curriculares del proceso de enseñanza aprendizaje.

d) Búsqueda, estudio sistemático, procesamiento y registro del contenido de los programas, los contenidos formativos transversales y de los valores sociales fundamentales a desarrollar en la enseñanza y su integración al proceso educativo.

e) Búsqueda, estudio sistemático, procesamiento y registro de la información política, histórica, económica, artística, científica y sus relaciones con el contenido de la educación de los tutorados.

f) Búsqueda, estudio sistemático, procesamiento y registro para su integración al proceso de educación de las tradiciones, potencialidades y problemáticas principales de los tutorados en los contextos del grupo, escuela, familia, comunidad, la nación y la humanidad. 
g) Búsqueda, estudio sistemático, procesamiento y registro para su utilización de la teoría y metodología de la investigación científica.

h) Búsqueda, estudio sistemático, procesamiento y registro para su utilización en el proceso educativo de los contenidos de la lengua materna, idiomas extranjeros, teoría de la comunicación, informática y la tecnología educativa.

\section{Competencia de diseño del proceso educativo.}

Se define como la formación psicológica de la personalidad y el constructo que designa la idoneidad del docente para planificar de forma creadora y contextualizada el proceso educativo y realizar la transposición didáctica del contenido de la cultura a la actividad de educación del tutorado. En esta competencia se destacan las acciones de diagnosticar, pronosticar, seleccionar y estructurar contenidos, seleccionar fuentes, determinar los métodos, procedimientos, tareas docentes, concebir la evaluación y las formas de organización, para los diferentes tipos de diseño. Se manifiesta en la capacidad metodológica.

\section{Competencia comunicativa-orientadora.}

Se define como la formación psicológica de la personalidad y el constructo que designa la idoneidad del docente para establecer todas las interrelaciones en el proceso de educación de los tutorados a través de las acciones de orientación, organización, control y evaluación de la educación de los tutorados en todas sus dimensiones y de su propia actividad pedagógica y se manifiesta en su desempeño a través de la capacidad comunicativa.

Integra cualidades y habilidades del tutor para establecer una adecuada relación interpersonal con el estudiante durante el proceso de tutoría; con una actitud abierta, inquisitiva y crítica para identificar sus intereses, sus motivaciones, sus fortalezas y debilidades académicas. Además de tener una actitud empática, dinámica y asertiva para estimular las ideas y propuestas que genere el estudiante en su proceso de formación hacia una vida académica independiente. 
Las competencias anteriores no son estáticas y cuando ocurran modificaciones a los programas académicos se tendrán que analizar y, en su caso, modificar y agregar las que sean necesarias para el logro del propósito fundamental del posgrado. Guardan estrecha relación con los roles del tutor analizados anteriormente. Asimismo, representan una primera aproximación teórica que deberá enriquecerse con el quehacer académico tutoral.

\section{La evaluación del desempeño del tutor.}

En América Latina muchos agentes educativos consideran que para que se generen necesidades de autoperfeccionamiento continuo de su gestión en el personal docente, resulta imprescindible que este se someta consciente y periódicamente a un proceso de evaluación de su desempeño.

Otros actores educativos, sin embargo, obstaculizan todo esfuerzo porque se instauren políticas de este tipo en sus sistemas educativos, a partir de posiciones básicamente gremiales que, tratando de "proteger al docente", olvidan el derecho de los alumnos a recibir una educación cualitativamente superior e incluso no reflexionan en el derecho que tienen los docentes a recibir acciones de asesoramiento y control que contribuyan al mejoramiento de su trabajo.

La evaluación profesoral no debe verse como una estrategia de vigilancia jerárquica que controla las actividades de los profesores, sino como una forma de fomentar $\mathrm{y}$ favorecer el perfeccionamiento del profesorado, como una manera de identificar las cualidades que conforman a un buen profesor para, a partir de ahí, generar políticas educativas que coadyuven a su generalización.

Por la función social que realizan los tutores están sometidos constantemente a una valoración por todos los que reciben directa o indirectamente sus servicios. Estas valoraciones y opiniones que se producen de forma espontánea sobre su comportamiento o competencia, e independientemente de la voluntad de los distintos factores que intervienen 
en el sistema escolar, pueden dar lugar a situaciones de ambiguiedad, a contradicciones, a un alto nivel de subjetivismo y, en ocasiones, pueden ser causa de decisiones inadecuadas y de insatisfacción y desmotivación de los docentes.

Por esa razón se hace necesario un sistema de evaluación que haga justo y racional ese proceso y que permita valorar su desempeño con objetividad, profundidad, e imparcialidad.

Las aproximaciones éticas y políticas a la evaluación subrayan que el problema más acuciante del proceso evaluador del profesor-tutor es el que atañe al empleo que la administración y la comunidad educativa puedan hacer de los informes o certificados de evaluación y de las implicaciones derivadas de ella. La evaluación puede utilizarse para impulsar la realización profesional, la autonomía y la colaboración entre los docentes, o bien puede invertirse y promover recelos, miedos y rechazo expreso del profesorado debido a las desviaciones de que pueda ser objeto la evaluación y sus consecuencias para los docentes.

La evaluación, en sí misma, ha de ser una opción de reflexión y de mejora de la realidad, pero su oportunidad y sentido de repercusión tanto en la personalidad del evaluado, como en su entorno y en el equipo del que forma parte, ha de ser entendida y situada adecuadamente para posibilitar el avance profesional de los docentes.

La evaluación del desempeño profesional del tutor es un proceso sistemático de obtención de datos válidos y fiables, con el objetivo de comprobar y valorar el efecto educativo que produce en los tutorados el despliegue de sus capacidades pedagógicas, su emocionalidad, responsabilidad laboral y la naturaleza de sus relaciones interpersonales con tutorados, padres, directivos, colegas y representantes de las instituciones sociales.

De la definición anterior se infiere que evaluar es proceder a conocer una realidad pasada, en su máxima extensión, destacando los conflictos en las 
condiciones y acciones realizadas, avanzando hipótesis de mejora y sobre todo, a partir del conjunto de datos e informes más fundamentados, con la máxima intervención de los participantes, emitir un juicio sobre la amplitud, evolución y complejidad de la tarea.

La evaluación es un juicio de valor que necesita referentes bien consolidados a los que tender y con los que contrastar la realidad evaluada, más esta constatación exigiría plena coincidencia en la identificación de tales referentes y en su aplicación.

La definición anterior nos proporciona una clave para la determinación de las dimensiones que debe comprender la operacionalización de la variable “desempeño profesional del tutor", y por lo tanto resulta muy útil para la construcción de una definición operacional del referido concepto.

\section{¿Cómo evaluar el desempeño docente?}

Para evaluar la calidad del desempeño docente se han utilizado distintas estrategias y fuentes de información, entre las que destacan:

- A través de los logros alcanzados por el tutorado.

- A través de instrumentos estandarizados que midan habilidad docente.

- A través de la opinión de los tutorados.

- Con base en la opinión de los tutores (autoevaluación).

- Con base en la opinión de los supervisores, directores y otras autoridades docentes.

- Evaluación por pares.

- Evaluación de la docencia mediante portafolios.

Cada una de ellas tiene características propias, ventajas y desventajas por lo que lo más adecuado sería evaluar empleando variedad de estrategias y fuentes. Cuando se cuenta con información sobre el desempeño de los tutores, los comités académicos pueden realizar acciones benéficas para 
la planta docente en su conjunto, porque a partir del diagnóstico que se desprende de la evaluación es posible identificar los puntos débiles y tratar de fortalecerlos. También es posible consolidar los puntos fuertes, al poner en marcha acciones ya probadas en diversos programas, comoel reconocimiento a la excelencia de los tutores

Propuesta de indicadores para evaluar la calidad del desempeño del tutor:

- El nivel de preparación alcanzado, que refleja un acervo acumulativo de la práctica pedagógica y del pensamiento humano en general, en constante perfeccionamiento dado el actual desarrollo permanente de la ciencia y la técnica, el entorno y las características del nivel de formación correspondiente.

- Nivel de desarrollo de sus competencias pedagógicas profesionales.

- Rol que desempeña en la dirección del proceso de formación profesional y personal de la personalidad de sus tutorados

- Actitud reflexiva sobre su práctica y la repercusión de esta en sus alumnos y en la sociedad.

Evaluar al tutor no es proyectar en él las deficiencias o razonables limitaciones del Sistema Educativo, sino es asumir un nuevo estilo, clima y horizonte de reflexión compartida para optimizar y posibilitar espacios reales de desarrollo profesional de los docentes, de generación de culturas innovadoras en los centros.

\section{Conclusiones}

En el actual contexto de exigencias investigativas y de producción académica en el contexto universitario es necesario desarrollar competencias investigativas, las cuales se caracterizan por ser complejas y requiere del apoyo tutorial.

Las competencias son características de las personas, pero no como cualidades abstractas de la personalidad, sino como realizaciones efectivas 
en una situación laboral, como un saber ser y saber hacer, por tal motivo el apoyo tutorial debe permitir e impulsar estos saberes.

El proceso de tutoría frecuentemente se concibe exclusivamente como una relación bipersonal, desde luego necesaria e indispensable, empero se ignora que la formación culmina cuando los novatos se transforman en expertos plenamente integrados tanto a comunidades de práctica (Wenger, 2001) como a redes sociales de expertos nacionales e internacionales. En consecuencia, la tutoría debe considerarse como un proceso dual, formativo y socializador; siendo indispensable generar nuevos modelos más abarcadores.

\section{Referencias}

ANUIES. (2000). Programas institucionales de tutoría. Una propuesta de la ANUIES para su organización y funcionamiento en las instituciones de educación superior. México: ANUIES.

Coromoto J. (2007). La superación continua del maestro tutor: una reflexión Oportuna de la Misión Sucre en el Estado Cojedes. Evento Internacional de Pedagogía. La Habana.

Ceja, C.M., Venegas, G.F.J., \& Armenta, A.M.A. (1998). Metodología para el diseño de un sistema de tutoría personalizada para tutorados del nivel superior en la Universidad de Colima. Material del Curso: Liderazgo y Gestión. Universidad de Colima, México.

Cruz G., García, T Y., \& Abreu, L.F (2006). Modelo Integrador de la Tutoría. De la dirección de tesis a la sociedad del conocimiento. Revista Mexicana de Investigación Educativa, 11(31), 1363-1388. Recuperado de:http://www.comie.org.mx/revista/v2018/rmie/index.php/nrmie/ article/view/738

Dicconario Enciclopédico Uteha. (1953). Tomo V. México.

Fuentes H., \& Cruz, S. (2001) Una concepción curricular para el siglo XXI, en respuesta a los retos de la sociedad y el conocimiento. Centro de Estudio de Educación Superior "Manuel F. Gran" Universidad de Oriente, Venezuela. 
Garritz, A., \& López, D.M. (1989). Tutoría: El perfil del docente en el posgrado, OMNIA, 1, 61-65

Latapí, S.P., (1988). La enseñanza tutorial: elementos para una propuesta orientada a elevar la calidad. Revista de la Educación Superior. Recuperado de:http://publicaciones.anuies.mx/revista/68/1/1/es/la-ensenanza-tutorialelementos-para-una-propuesta-orientada-a-elevar

Martínez, M. (2005). El Profesor Tutor en el proceso de Universalización de la Educación Superior. Evento Internacional de Pedagogía. La Habana, Cuba.

Merton, K. (1973). Hacia la codificación del análisis funcional. En: El funcionalismo en la sociología norteamericana. Editora Política. La Habana,

Parsons, T. (1945). Teoría de la acción social. Chicago.

Sánchez, F. (2001). Las tutorías y la construcción de ambientes de aprendizaje en la educación abierta y a distancia. Recuperado de: http://www.cifiu. uni.edu.ni/docs/coordinadores/pdf/t_3_lec_2.pdf

Sarukhán, J. (1988). La tutoría en la enseñanza universitaria, OMNIA, 1, 5-8.

Stehr, N. (1994). Knowledge societies, Londres: SAGE Publications.

Wenger, E. (2001). Comunidades de práctica: aprendizaje, significado e identidad. Barcelona: Paidós. 